\title{
Diagnostic Performance of Deep Learnlng AlgoriThm for Analysis of Computed Tomography Myocardial PerfusION
}

\section{Giuseppe Muscogiuri}

Centro Cardiologico Monzino IRCCS: Centro Cardiologico Monzino Istituto di Ricovero e Cura a Carattere Scientifico

\section{Mattia Chiesa}

Monzino Cardiology Centre: Centro Cardiologico Monzino Istituto di Ricovero e Cura a Carattere

Scientifico

\section{Andrea Baggiano}

Monzino Cardiology Centre: Centro Cardiologico Monzino Istituto di Ricovero e Cura a Carattere

Scientifico

\section{Pierino Spadafora}

Università degli Studi di Milano: Universita degli Studi di Milano

\section{Rossella De Santis}

University of Milan: Universita degli Studi di Milano

\section{Marco Guglielmo}

Centro Cardiologico Monzino IRCCS: Centro Cardiologico Monzino Istituto di Ricovero e Cura a Carattere Scientifico

\section{Stefano Scafuri}

Centro Cardiologico Monzino IRCCS: Centro Cardiologico Monzino Istituto di Ricovero e Cura a Carattere Scientifico

\section{Laura Fusini}

Centro Cardiologico Monzino IRCCS: Centro Cardiologico Monzino Istituto di Ricovero e Cura a Carattere Scientifico

\section{Saima Mushtaq}

Centro Cardiologico Monzino IRCCS: Centro Cardiologico Monzino Istituto di Ricovero e Cura a Carattere Scientifico

\section{Edoardo Conte}

Centro Cardiologico Monzino IRCCS: Centro Cardiologico Monzino Istituto di Ricovero e Cura a Carattere Scientifico

\section{Andrea Annoni}

Centro Cardiologico Monzino IRCCS: Centro Cardiologico Monzino Istituto di Ricovero e Cura a Carattere Scientifico 


\section{Alberto Formenti}

Centro Cardiologico Monzino IRCCS: Centro Cardiologico Monzino Istituto di Ricovero e Cura a Carattere Scientifico

\section{Maria Elisabetta Mancini}

Centro Cardiologico Monzino IRCCS: Centro Cardiologico Monzino Istituto di Ricovero e Cura a Carattere Scientifico

\section{Francesca Ricci}

Centro Cardiologico Monzino IRCCS: Centro Cardiologico Monzino Istituto di Ricovero e Cura a Carattere Scientifico

\section{Francesco Paolo Ariano}

Centro Cardiologico Monzino IRCCS: Centro Cardiologico Monzino Istituto di Ricovero e Cura a Carattere Scientifico

\section{Luigi Spiritigliozzi}

Centro Cardiologico Monzino IRCCS: Centro Cardiologico Monzino Istituto di Ricovero e Cura a Carattere Scientifico

\section{Mario Babbaro}

Centro Cardiologico Monzino IRCCS: Centro Cardiologico Monzino Istituto di Ricovero e Cura a Carattere Scientifico

\section{Rocco Mollace}

Centro Cardiologico Monzino IRCCS: Centro Cardiologico Monzino Istituto di Ricovero e Cura a Carattere Scientifico

\section{Riccardo Maragna}

Centro Cardiologico Monzino IRCCS: Centro Cardiologico Monzino Istituto di Ricovero e Cura a Carattere Scientifico

\section{Carlo Maria Giacari}

Centro Cardiologico Monzino IRCCS: Centro Cardiologico Monzino Istituto di Ricovero e Cura a Carattere Scientifico

\section{Daniele Andreini}

Centro Cardiologico Monzino IRCCS: Centro Cardiologico Monzino Istituto di Ricovero e Cura a Carattere Scientifico

\section{Andrea Igoren Guaricci}

Azienda Ospedaliero-Universitaria Consorziale Policlinico di Bari

\section{Gualtiero I. Colombo}

Centro Cardiologico Monzino IRCCS: Centro Cardiologico Monzino Istituto di Ricovero e Cura a Carattere Scientifico

\section{Mark G. Rabbat}

Loyola University Chicago

\section{Mauro Pepi}

Centro Cardiologico Monzino IRCCS: Centro Cardiologico Monzino Istituto di Ricovero e Cura a Carattere Scientifico 


\section{Francesco Sardanelli}

University of Milan: Universita degli Studi di Milano

Gianluca Pontone ( $\nabla$ gianluca.pontone@ccfm.it)

Centro Cardiologico Monzino IRCCS https://orcid.org/0000-0002-1339-6679

\section{Research Article}

Keywords: myocardial CT perfusion, convolutional neural network, coronary artery disease, coronary computed tomography angiography, myocardial ischemia

Posted Date: November 3rd, 2021

DOI: https://doi.org/10.21203/rs.3.rs-1037278/v1

License: (c) (i) This work is licensed under a Creative Commons Attribution 4.0 International License.

Read Full License 


\section{Abstract}

Purpose: Artificial intelligence could play a key role in cardiac imaging analysis. To evaluate the diagnostic accuracy of a deep learning (DL) algorithm predicting hemodynamically significant coronary artery disease (CAD) by using a rest dataset of myocardial computed tomography perfusion (CTP) as compared to invasive evaluation.

Methods: One hundred and twelve consecutive symptomatic patients scheduled for clinically indicated invasive coronary angiography (ICA) underwent CCTA plus static stress CTP and ICA with invasive fractional flow reserve (FFR) for stenoses ranging between $30 \%$ and $80 \%$. Subsequently, a DL algorithm for the prediction of significant CAD by using the rest dataset (CTP-DL $L_{\text {rest }}$ ) and stress dataset (CTP$\left.D L_{\text {stress }}\right)$ was developed. The diagnostic accuracy for identification of significant CAD using CCTA,

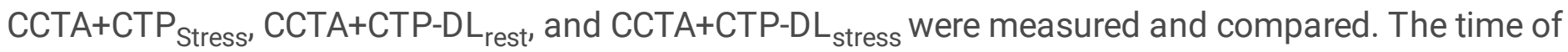
analysis for $\mathrm{CTP}_{\text {Stress, }}$ CTP-DL $\mathrm{L}_{\text {rest }}$ and CTP-DL $\mathrm{L}_{\text {Stress }}$ were recorded.

Results: Patient-specific sensitivity, specificity, NPV, PPV, accuracy and area under the curve (AUC) of CCTA alone and CCTA+CTP Stress $_{\text {w }}$ were $100 \%, 33 \%, 100 \%, 54 \%, 63 \%, 67 \%$ and $86 \%, 89 \%, 89 \%, 86 \%, 88 \%$, $87 \%$, respectively. Patient-specific sensitivity, specificity, NPV, PPV, accuracy and AUC of CCTA+DL rest and

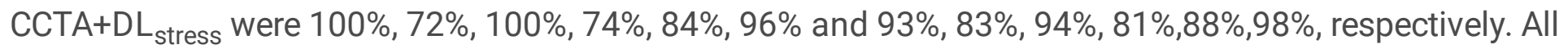
CCTA+CTP Stress, CCTA+CTP-D $L_{\text {Rest }}$ and CCTA+CTP-DL $L_{\text {Stress }}$ significantly improved detection of hemodynamically significant CAD $(p<0.01)$.

Time of CTP-DL was significantly lower as compared to human analysis $(39.2 \pm 3.2$ vs. $379.6 \pm 68.0$ seconds, $\mathrm{p}<0.001)$.

Conclusion: Evaluation of myocardial ischemia using a DL approach on rest CTP datasets is feasible and accurate. This approach may be a useful gatekeeper prior to CTP $_{\text {Stress. }}$

\section{Introduction}

Coronary artery disease (CAD) represents one of the leading causes of mortality in the USA [1].

Traditionally, anatomical evaluation of coronaries was strictly confined to invasive coronary angiography (ICA); today, coronary computed tomography angiography (CCTA) represents an important tool to rule out CAD $[2,3]$.

Novel techniques have developed such as CT myocardial perfusion under stress conditions $\left(\right.$ CTP $\left._{\text {Stress }}\right)$ and fractional flow reserve computed tomography derived (FFR $\mathrm{CT}_{\mathrm{CT}}$ ) demonstrating the possibility to bridge the coronary anatomy with the physiology and evaluate myocardial ischemia $[4,5]$.

The applications of $\mathrm{FFR}_{\mathrm{CT}}$ and $\mathrm{CTP}_{\text {Stress }}$ are not widely applied in clinical practice. Ideally, $\mathrm{CTP}_{\text {Stress }}$ uses image datasets acquired with last generation CT scanners [6] that are not available in all centers and the 
readers need specific training in order to avoid inaccurate interpretation of images $[7,8]$. In addition, CTP $_{\text {Stress }}$ requires a double acquisition (rest and stress) with related iodine contrast agent administration, radiation exposure concerns and the use of a stressor with potential side effects $[4,9]$.

Application of artificial intelligence in cardiac imaging is ranging from image acquisition to image reporting and prognostic stratification [10-13]. An advantage of this approach was highlighted by the possibility to speed up the time of reporting [10].

The aim of this article is to show the diagnostic accuracy of a deep learning (DL) model trained on rest and stress CTP for the identification of reversible myocardial ischemia compared with CCTA+CTP Stress $_{\text {s }}$ using ICA plus clinically indicated invasive FFR as the reference standard.

\section{Methods}

\section{Population}

One hundred and forty-seven consecutive patients with suspected CAD enrolled in the PERFECTION study $[14,15]$ were retrospectively analyzed for this study. Patients with a history of previous myocardial infarction, acute coronary syndrome, previous revascularization, impaired renal function or contraindication to administration of contrast agent, pregnancy, cardiac arrhythmias, inability to sustain breath hold, BMl> 35 and contraindication to administer B-blockers and nitrates were excluded from the study as previously described $[14,15]$. The institutional ethical committee approved the protocol and all patients signed an informed consent.

\section{Images Acquisition \\ Patient Preparation}

Patients were asked to refrain from caffeine and smoking for 24 hours and fasting 6 hours prior the CTCA examination.

During rest CCTA, in patients with heart rate $>65$ beats $/ \mathrm{min}(\mathrm{bpm})$, metoprolol with a titration dose up to $15 \mathrm{mg}$ was administered intravenously in order to obtain a HR $\leq 65 \mathrm{bpm}$.

Before the rest scan, all patients received sublingual nitrates to ensure coronary vasodilatation.

\section{CCTA}

All examinations were performed using a Revolution CT scanner (GE Healthcare, Milwaukee, Wisconsin) following the guidelines of the Society of Cardiovascular Computed Tomography (SCCT)[16]. The Rest CCTA parameters have been described previously [5]. 
Based on HR, CCTA was acquired in 70-80\% of the cardiac cycle in patients with HR $\leq 65 \mathrm{bpm}$, while in patients with $>65 \mathrm{bpm}$, CCTA was acquired between $40-80 \%$ of cardiac cycle. CCTA images were acquired after the injection of $70 \mathrm{ml}$ of iodine contrast agent (Visipaque $320 \mathrm{mg} / \mathrm{ml}$ ) at $6.2 \mathrm{ml} / \mathrm{sec}$ followed by $50 \mathrm{ml}$ of saline at the same flow rate of contrast agent.

All scans were performed using the bolus tracking technique with visual assessment to determine the correct timing for acquisition.

All images were reconstructed using an adaptative statistical iterative reconstruction (ASIR-V, GE Healthcare, Milwaukee, Wisconsin)[17]. Datasets of CCTA were transferred to an image-processing workstation (Advantage Workstation 4.7, GE Healthcare, Milwaukee, Wisconsin) to perform quantitative coronary analysis according to SCCT guidelines for reporting by two certified expert readers with more than 8 years of experience in cardiovascular imaging following the European Association Cardiovascular Imaging (EACVI) guidelines for training and certification [18]. In case of disagreement, a third cardiac radiologist evaluated the images.

\section{CTP $_{\text {Stress }}$}

The patient underwent intravenous administration of adenosine $(0.14 \mathrm{mg} / \mathrm{Kg} / \mathrm{min})$ over 4 minutes. At the end of the third minute of adenosine administration, a CCTA was acquired using the same technical parameters previously described [5]. Subsequently, images were transferred to an offline workstation (Advantage Workstation Version 4.7, GE Healthcare), reconstructed on short axis and long axis with an average slice thickness between 4-8mm using a narrow window width and level of $350 \mathrm{~W}$ and $150 \mathrm{~L}$, respectively. Perfusion datasets were analyzed in consensus by two certified expert readers, blinded to clinical history and CCTA findings, with more than 8 years of experience in cardiovascular imaging following the EACVI guidelines for training and certification [18]. In case of disagreement, a third reader evaluated the images.

\section{Combined CCTA+CTP Stress interpretation}

Coronary arteries at CCTA were segmented according to the American Heart Association (AHA) model [19]. Obstructive CAD was defined as coronary stenosis $>50 \%$.

CTP $_{\text {Stress }}$ was evaluated according to the AHA myocardial segmentation [20] model. Stress positivity was defined as any subendocardial hypo-enhancement extending more than $25 \%$ of transmurality in a specific territory that was not present at rest CCTA.

Matching between CCTA and CTP Stress $_{\text {findings was performed according to the algorithm previously }}$ described $[14,20,21]$.

Briefly, the adjudication process was applied each time there was a coronary arterial lesion with $>50 \%$ diameter stenosis and at least 1 myocardial perfusion defect in the matched myocardial segment. 
Time of analysis of image reporting for CTP $_{\text {Stress }}$ was recorded.

\section{ICA and invasive FFR performance and interpretation}

All patients underwent ICA following the guidelines of the American College of Cardiology/AHA Task Force on Practice Guidelines and the Society for Cardiac Angiography and Interventions [22]. The coronary arteries were classified according to the AHA Classification system [19]. An interventional cardiologist blinded to results of CCTA analyzed ICA images with quantitative coronary angiography (QCA) (QantCor QCA; Pie Medical Imaging, Maastricht, the Netherlands).

Coronary stenosis was evaluated calculating the percentage of narrowing using the minimum diameter and reference diameter. In the presence of stenosis ranging between $30 \%$ and $80 \%$, invasive FFR was calculated [23]. In order to calculate FFR, the pressure wire (Certus Pressure Wire, St. Jude Medical Systems, St. Paul, MI) was equalized with the aortic pressure and subsequently was placed distal to the stenosis in the distal third of the coronary artery with stenosis. An injection of $100 \mathrm{mg}$ of glyceryl trinitrate was injected intracoronary in order to prevent vasospasm while $140 \mathrm{mg} / \mathrm{Kg} / \mathrm{min}$ of adenosine was administered intravenously in order to create an inducible stress. FFR was assessed the peak of hyperemia using the RadiAnalyzer Xpress (Radi Medical Systems, Uppsala, Sweden) by dividing the mean coronary pressure, measured with the pressure sensor placed distal to the stenosis, by the mean aortic pressure measured through the guide catheter. Intermediate stenoses showing values of invasive FFR $\leq 0.8$ or anatomical stenoses showing $>80 \%$ diameter reduction or total occlusions were considered functionally significant.

\section{Deep Learning analysis}

\section{Dataset generation}

We selected 112 patients from the 147 studied; 58 with invasive FFR $\leq 0.8$ or stenosis $>80 \%$ (Group 0 ) and 54 with invasive FFR $>0.8$ (Group 1). Thirty five patients were excluded due to poor image quality or an inconsistent dataset. First, we randomly split the dataset in two subsets: an "independent test set", generated selecting 18 and 14 samples from Group 0 and Group 1, respectively, and a "learning set" composed of the remaining 80 . To limit the effect of overfitting during the training step, a data augmentation step was applied. At the end, the learning set consisted of 80 group 0 and 80 group 1 samples. The learning set was used to train the algorithms and to identify its best configuration of parameters and hyper-parameters; conversely, the independent test set was used to assess the performance.

\section{DICOM-to-Image pre-processing}

Each dataset (rest and stress) scan was composed of 256 or 224 slices ( $512 \times 512$ pixels) and stored as a DICOM. To focus our attention on the left ventricle, we manually defined a region of interest (a 250-by$250 \mathrm{px}$ window) around the left ventricle, allowing a decrease of computational burden and improving the 
overall network performance. Finally, the resulting slices were joined in a 10-by-10 squared image, resized to $1280 \times 1280$ pixels.

\section{Convolutional neural network (CNN) architecture}

The keras package has been used to build and test the CNN. The convolutional section of the network consisted of four consecutive layer blocks, each one containing a single convolutional layer and a maxpooling layer. A densely connected network with 4 hidden layers (512, 256, 64 and 32 neurons) and the output layer followed the convolutional section. To handle overfitting a dropout strategy was implemented before the first hidden layer (dropout rate: 0.2) [24]. The ReLU activation function[25] was used for each neuron (densely connected and convolutional ones), except for the output node (activation function: 'sigmoid'). Finally, we set the 'Stochastic Gradient Descend' optimizer to minimize the 'binary cross entropy' loss function, the number of max epochs $=500$ and the batch size $=20$.

The CNN was trained in order to predict the ischemic myocardium by using both rest (CTP-DL Rest $)$ and stress (CTP-DL $\left.L_{\text {Stress }}\right)$ datasets based on the values of invasive FFR. The time of analysis for the evaluation of algorithms for both CTP-DL ${ }_{\text {Rest }}$ and CTP-DL $L_{\text {Stress }}$ were recorded.

\section{Statistical Analysis}

Statistical analysis was performed using SPSS version 21.0 software (SPSS, Chicago, Illinois) and R version 4.0.2 (R Foundation for statistical Computing, Vienna, Austria).

Continuous variables are presented as means \pm SD, while categorical data are reported as frequencies and percentages and compared with T-test Student or Chi-squared test, respectively. Association between variables were assessed by Pearson's correlation coefficient (R).

Patient-based sensitivity, specificity, negative predictive value (NPV), positive predictive value (PPV), diagnostic accuracy and area under the curve (AUC) were measured for CCTA, CCTA+CTP Stress, $_{\text {, }}$ CCTA+CTP-DL $L_{\text {Rest }}$ and CCTA+CTP-DL $L_{\text {Stress }}$ on the independent test set and DeLong method used to compare the differences in terms of AUC between all approaches.

Time of analysis between human and DL algorithm for both CTP-DL $L_{\text {rest }}$ and CTP-DL $L_{\text {Stress }}$ were compared. Differences were deemed significant if the $p$-value was $<0.05$.

\section{Results}

Baseline characteristics of the overall population are summarized in Table 1. Obstructive CAD was observed in 78 out of 112 patients (66\%) while the prevalence of functionally significant CAD was detected in 54 out of 112 patients (49\%). CTP Stress, $_{\text {CTP-DL }}$ Rest and CTP-DL $L_{\text {Stress }}$ were successfully performed in all patients.

Table 1 Characteristics of the Study Population 
The sensitivity, specificity, NPV, PPV, diagnostic accuracy and AUC of CCTA alone and CCTA+CTP in order to detect significant CAD were $100 \%, 33 \%, 100 \%, 54 \%, 63 \%, 67 \%$ and $86 \%, 89 \%, 89 \%, 86 \%, 88 \%, 87 \%$, respectively (Table 2 and Figure 1). Combining the anatomical data of CCTA and the CTP-DL applied to rest and stress datasets, we observed a sensitivity, specificity, NPV, PPV, diagnostic accuracy and AUC of $100 \%, 72 \%, 100 \%, 74 \%, 84 \%, 96 \%$ and $93 \%, 83 \%, 94 \%, 81 \%, 88 \%, 98 \%$ for CCTA+CTP-DL rest $_{\text {and }}$ CCTA+CTP-DL stress, $_{\text {, }}$ respectively (Table 2 and Figure 1 ).

Table 2. Diagnostic accuracy of CCTA, CCTA+CTP, DL Rest, DL Stress, CCTA+DL rest and CCTA+DL stress for the evaluation of significant CAD using ICA+FFR as the reference standard

\section{CCTA CCTA+Stress CTP CCTA+CTP-DL rest $\quad$ CCTA+CTP-DL stress}

\begin{tabular}{lllll}
\hline Sens & $100 \%(77 \%-100 \%)$ & $86 \%(57 \%-98 \%)$ & $100 \%(77 \%-100 \%)$ & $93 \%(66 \%-100 \%)$ \\
\hline Spec & $33 \%(13 \%-59 \%)$ & $89 \%(65 \%-99 \%)$ & $72 \%(47 \%-90 \%)$ & $83 \%(59 \%-96 \%)$ \\
\hline NPV & $100 \%(100 \%-100 \%)$ & $89 \%(69 \%-97 \%)$ & $100 \%(100 \%-100 \%)$ & $94 \%(69 \%-99 \%)$ \\
\hline PPV & $54 \%(46 \%-62 \%)$ & $86 \%(62 \%-96 \%)$ & $74 \%(57 \%-86 \%)$ & $81 \%(60 \%-92 \%)$ \\
\hline AcC & $63 \%(44 \%-79 \%)$ & $88 \%(71 \%-97 \%)$ & $84 \%(67 \%-95 \%)$ & $88 \%(71 \%-97 \%)$ \\
\hline AUC & $67 \%(55 \%-78)$ & $87 \%(75 \%-99 \%)$ & $96 \%(91 \%-100 \%)$ & $98 \%(94-100 \%)$ \\
\hline
\end{tabular}

Values are expressed as $\mathrm{Cl}$ of 95\%. AUC: Area Under Curve; Acc: Accuracy; CCTA: Coronary Computed Tomography Angiography; CTP: CT perfusion; DL rest: Deep learning rest perfusion; DL stress: Deep learning stress perfusion; FFR: Fractional flow reserve; ICA: Invasive coronary angiography; Sens: sensitivity; Spec: Specificity; NPV: Negative predictive value; PPV: positive predictive value

The DL algorithm was associated with a correct prediction regardless of the vessel involved in stress and rest datasets, while a significant but fair correlation observed with transmurality in the stress dataset. If the significant pathology was located in the LAD and RCA the algorithm showed better prediction when compared to the LCX (Figure 2).

The evaluation of myocardial perfusion using DL was significantly lower as compared to the standard human approach (39.2 \pm 3.2 vs. $379.6 \pm 68.0$ seconds, $p<0.001)$ (Table 3 ). Representative cases are shown in Figures 3 and 4.

Table 3. Associations of vascular territory and transmurality with the DL approach. 


\section{Demographic Characteristics}

Age (years)

Men

BMI $(\mathrm{Kg} / \mathrm{m} 2)$

Risk factors

Hypertension

Smoker

Hyperlipidemia

Diabetes

Family history

Symptoms

Atypical angina

Typical angina

Pre-test likelihood of CAD

No previous testing

Positive-exercise ecg

Positive stress echocardiography

Positive SPECT

Positive stress MRI

\section{Rest scan protocol}

HR before scanning, beats/min

Beta-blocker

Beta-blocker dosage (mg)

HR during scanning, beats/min

Dose length product, $\mathrm{mGy} \mathrm{x} \mathrm{cm}$

Effective dose, mSv

Stressscan protocol

HR during scanning, beats/min

Dose length product, $\mathrm{mGy} \mathrm{x} \mathrm{cm}$
$66.58 \pm 9$

$81(73 \%)$

$26.5 \pm 3.7$

$85(75 \%)$

$28(25 \%)$

40 (35\%)

$20(17 \%)$

$67(59 \%)$

$52(46 \%)$

60 (54\%)

$67,29 \pm 12,3$

$31(27 \%)$

$51(45,5 \%)$

$6(5 \%)$

$21(18 \%)$

$3(2 \%)$

$68 \pm 11$

$58(50 \%)$

$5.22 \pm 6.4$

$62 \pm 9$

$195.59 \pm 100.9$

$2.74 \pm 1.4$ 
Effective dose, mSv

$2.51 \pm 0.9$

\section{Prevalence of significant CAD at ICA+FFR}

Absence of obstructive CAD

1-vessel disease

2-vessel disease

3-vessel disease

Absence of functionally significant CAD

1-vessel disease

2-vessel disease

3-vessel disease
$34(30 \%)$

$41(37 \%)$

$15(14 \%)$

$22(19 \%)$

$58(51 \%)$

$25(23 \%)$

$13(12 \%)$

$16(14 \%)$

Values are expressed as mean $\pm S D$ or absolute number and percentage

BMI: body mass index; CAD: coronary artery disease; CCTA: coronary computed tomography angiography; FFR: fractional flow reserve; ICA: invasive coronary angiography; MRI: magnetic resonance imaging; SPECT: single photon emission computed tomography.

\begin{tabular}{|llll|}
\hline & DL Stress & & \\
\hline & $\mathbf{R}$ & $\mathbf{R}^{2}$ & p-value \\
\hline LAD & 0.56 & 0.31 & 0.0009 \\
\hline LCX & 0.44 & 0.19 & 0.01 \\
\hline RCA & 0.61 & 0.37 & 0.0002 \\
\hline Transmurality & 0.36 & 0.13 & 0.038 \\
\hline LAD & DL Rest & & 0.0011 \\
\hline LCX & 0.55 & 0.30 & 0.035 \\
\hline RCA & 0.37 & 0.14 & 0.003 \\
\hline $\begin{array}{l}\text { DL: Deep learning; LAD: Left anterior descending artery; LCX: Left circumflex artery; RCA: Right } \\
\text { Coronary artery }\end{array}$ & 0.51 & 0.26 \\
\hline
\end{tabular}

\section{Discussion}

In this paper, we showed the possibility to provide information regarding ischemic myocardium using a fully automated $D L$ algorithm with comparable diagnostic accuracy and shorter time to human analysis. The DL algorithm can be applied to both rest and stress datasets with similar high diagnostic accuracy. 
The clinical application of these findings can be further improved with the combination of anatomical evaluation and myocardial perfusion using the DL analysis with an AUC, diagnostic accuracy and negative predictive value of $96 \%, 84 \%$ and $100 \%$, respectively. Irrespective of the vessel involved and transmurality, for stress datasets, the DL algorithm seems to provide good performance.

Another important finding was demonstrated by the short time of analysis of myocardium using a DL approach.

Few papers in the literature analyzed CTP using an artificial intelligence approach [26, 27].

Xiong et al developed a machine learning algorithm that was able to identify myocardial ischemia on rest images [26]. The authors developed an algorithm that was helpful in providing the myocardial contours and identify the myocardial segments susceptible for myocardial ischemia when compared with invasive quantitative coronary angiography [26]. Despite the authors lack of a gold standard for detection of myocardial ischemia, they developed an algorithm that was able to detect impairment of myocardial perfusion in patient with significant stenosis with an accuracy, sensitivity and specificity of $70 \%, 79 \%$ and $64 \%$. However, it is important to consider that the authors did not evaluate the presence of ischemia with invasive FFR. Moreover, the analysis of rest perfusion using the DL approach was time consuming considering the post-processing of images.

Another technique able to identify a rest perfusion defect was developed by Van Hamersvelt et al. [27]. The authors developed a DL algorithm that was able to identify the presence of myocardial ischemia assessed by invasive FFR from a rest CCTA. Similar to our article, the authors reported an increase in accuracy when the DL algorithm was combined with anatomical evaluation, AUC of $76 \%$ compared to $68 \%$ for anatomical evaluation alone [27]. Despite a robust gold standard represented by invasive FFR for the training of the DL algorithm, the authors did not evaluate the impact of their algorithm in a dataset of stress CTP. Furthermore, the analysis of perfusion using the DL approach required the segmentation of myocardium[27], while in our manuscript, it was not performed.

In our manuscript, beyond the feasibility of a new DL approach, we showed that is possible to provide better diagnostic accuracy compared to humans if the myocardium is analyzed with a DL technique in both rest and stress datasets. Furthermore, in our approach, it was possible to identify significant CAD with a fully automated technique. Importantly, the comparison between the human approach and the DL algorithm showed similar accuracy despite the time of analysis with the $\mathrm{DL}$ approach being $<1$ minute.

Following the ESC guidelines we hypothesize that the application of CCTA will exponentially grow [3].Therefore, the application of Al algorithms in clinical practice could speed up the time of acquisition, reporting and interpretation of images. A fully automated approach would be feasible in clinical practice with the first step being the evaluation of coronary arteries as shown by Muscogiuri et al [10] followed by evaluation of ischemia with a DL algorithm that could evaluate the myocardium. In particular, the evaluation of myocardium on rest CCTA is performed without the administration of a stressor agent allowing for a safe and cheap approach. 
Furthermore, the application of a DL algorithm that could identify myocardial ischemia on CCTA can potentially break down the costs of ischemia testing following CCTA. This algorithm could potentially run on a standard personal computer without any particular imaging skills.

Some limitations need to be addressed. First, the small sample size and the retrospective nature of the study represents a limitation of the study. Furthermore, the prevalence of CAD in the population examined is higher than expected in a normal population.

Second, a prospective larger study is needed in order to evaluate the prognostic impact of a CCTA+CTPDL rest approach.

In conclusion, evaluation of myocardial ischemia using a DL approach on rest CTP datasets is feasible and accurate. This approach may be a useful gatekeeper prior to further testing such as CTP

\section{References}

1. Ford ES, Capewell S. Coronary heart disease mortality among young adults in the U.S. from 1980 through 2002: concealed leveling of mortality rates. J Am Coll Cardiol. 2007;50:2128-32.

2. Pontone G, Andreini D, Bartorelli AL, Bertella E, Cortinovis S, Mushtaq S, et al. A long-term prognostic value of $C T$ angiography and exercise ECG in patients with suspected CAD. JACC CardiovasC Imaging. 2013;6:641-50.

3. Knuuti J, Wijns W, Saraste A, Capodanno D, Barbato E, Funck-Brentano C, et al. 2019 ESC Guidelines for the diagnosis and management of chronic coronary syndromes. Eur Heart J. 2020;41:407-77.

4. Pontone G, Muscogiuri G, Andreini D, Guaricci Al, Guglielmo M, Mushtaq S, et al. The New Frontier of Cardiac Computed Tomography Angiography: Fractional Flow Reserve and Stress Myocardial Perfusion. Curr Treat Options Cardiovasc Med. 2016;18:74.

5. Pontone G, Andreini D, Guaricci Al, Baggiano A, Fazzari F, Guglielmo M, et al. Incremental Diagnostic Value of Stress Computed Tomography Myocardial Perfusion With Whole-Heart Coverage CT Scanner in Intermediate- to High-Risk Symptomatic Patients Suspected of Coronary Artery Disease. JACC Cardiovasc Imaging. 2019;12:338-49.

6. Pontone G, Weir-McCall JR, Baggiano A, Del Torto A, Fusini L, Guglielmo M, et al. Determinants of Rejection Rate for Coronary CT Angiography Fractional Flow Reserve Analysis. Radiology. 2019;292:597-605.

7. Carrascosa P, Capunay C. Myocardial CT perfusion imaging for ischemia detection. Cardiovasc Diagn Ther. 2017;7:112-28.

8. Lee JM, Choi G, Koo BK, Hwang D, Park J, Zhang J, et al. Identification of High-Risk Plaques Destined to Cause Acute Coronary Syndrome Using Coronary Computed Tomographic Angiography and Computational Fluid Dynamics. JACC Cardiovasc Imaging. 2019;12:1032-43.

9. Kim SM, Cho YK, Choe YH. Adenosine-stress dynamic myocardial perfusion imaging using 128-slice dual-source CT in patients with normal body mass indices: effect of tube voltage, tube current, and 
iodine concentration on image quality and radiation dose. Int J Cardiovasc Imaging. 2014;30 Suppl 2:95-103.

10. Muscogiuri G, Chiesa M, Trotta M, Gatti M, Palmisano V, Dell'Aversana S, et al. Performance of a deep learning algorithm for the evaluation of CAD-RADS classification with CCTA. Atherosclerosis. 2020;294:25-32.

11. Al'Aref SJ, Anchouche K, Singh G, Slomka PJ, Kolli KK, Kumar A, et al. Clinical applications of machine learning in cardiovascular disease and its relevance to cardiac imaging. Eur Heart J. 2018.

12. Muscogiuri G, Van Assen $M$, Tesche $C$, De Cecco CN, Chiesa M, Scafuri S, et al. Artificial Intelligence in Coronary Computed Tomography Angiography: From Anatomy to Prognosis. Biomed Res Int. 2020;2020:6649410.

13. van Assen M, Muscogiuri G, Caruso D, Lee SJ, Laghi A, De Cecco CN. Artificial intelligence in cardiac radiology. Radiol Med. 2020;125:1186-99.

14. Pontone G, Andreini D, Guaricci Al, Guglielmo M, Mushtaq S, Baggiano A, et al. Rationale and design of the PERFECTION (comparison between stress cardiac computed tomography PERfusion versus Fractional flow rEserve measured by Computed Tomography angiography In the evaluation of suspected cOroNary artery disease) prospective study. J Cardiovasc Comput Tomogr. 2016;10:3304.

15. Pontone G, Baggiano A, Andreini D, Guaricci Al, Guglielmo M, Muscogiuri G, et al. Stress Computed Tomography Perfusion Versus Fractional Flow Reserve CT Derived in Suspected Coronary Artery Disease: The PERFECTION Study. JACC Cardiovasc Imaging. 2019;12:1487-97.

16. Abbara S, Blanke P, Maroules CD, Cheezum M, Choi AD, Han BK, et al. SCCT guidelines for the performance and acquisition of coronary computed tomographic angiography: A report of the society of Cardiovascular Computed Tomography Guidelines Committee: Endorsed by the North American Society for Cardiovascular Imaging (NASCI). J Cardiovasc Comput Tomogr. 2016;10:43549.

17. Pontone G, Muscogiuri G, Andreini D, Guaricci Al, Guglielmo M, Baggiano A, et al. Impact of a New Adaptive Statistical Iterative Reconstruction (ASIR)-V Algorithm on Image Quality in Coronary Computed Tomography Angiography. Acad Radiol. 2018;25:1305-13.

18. Pontone G, Moharem-Elgamal S, Maurovich-Horvat P, Gaemperli O, Pugliese F, Westwood M, et al. Training in cardiac computed tomography: EACVI certification process. Eur Heart J Cardiovasc Imaging. 2018;19:123-6.

19. Austen WG, Edwards JE, Frye RL, Gensini GG, Gott VL, Griffith LS, et al. A reporting system on patients evaluated for coronary artery disease. Report of the Ad Hoc Committee for Grading of Coronary Artery Disease, Council on Cardiovascular Surgery, American Heart Association. Circulation. 1975;51:5-40.

20. Cerqueira MD, Weissman NJ, Dilsizian V, Jacobs AK, Kaul S, Laskey WK, et al. Standardized myocardial segmentation and nomenclature for tomographic imaging of the heart. A statement for 
healthcare professionals from the Cardiac Imaging Committee of the Council on Clinical Cardiology of the American Heart Association. Circulation. 2002;105:539-42.

21. Cerci RJ, Arbab-Zadeh A, George RT, Miller JM, Vavere AL, Mehra V, et al. Aligning coronary anatomy and myocardial perfusion territories: an algorithm for the CORE320 multicenter study. Circ Cardiovasc Imaging. 2012;5:587-95.

22. Levine GN, Bates ER, Blankenship JC, Bailey SR, Bittl JA, Cercek B, et al. 2011 ACCF/AHA/SCAI Guideline for Percutaneous Coronary Intervention. A report of the American College of Cardiology Foundation/American Heart Association Task Force on Practice Guidelines and the Society for Cardiovascular Angiography and Interventions. J Am Coll Cardiol. 2011;58:e44-122.

23. Tonino PA, De Bruyne B, Pijls NH, Siebert U, Ikeno F, van't Veer M, et al. Fractional flow reserve versus angiography for guiding percutaneous coronary intervention. N Engl J Med. 2009;360:213-24.

24. Svrivastava N, Hinton G, Krizhevsky A, Sutskever I, Salakhutdinov R. Dropout: a simple way to prevent neural networks from overfitting. The Journal of Machine Learning Research. 2014.

25. Nair V, Hinton G. Rectified linear units improve restricted Boltzmann machines. In Proceedings of the 27th International Conference on Machine Learning-New York: ACM. 2010:807-14.

26. Xiong G, Kola D, Heo R, Elmore K, Cho I, Min JK. Myocardial perfusion analysis in cardiac computed tomography angiographic images at rest. Med Image Anal. 2015;24:77-89.

27. van Hamersvelt RW, Zreik M, Voskuil M, Viergever MA, Isgum I, Leiner T. Deep learning analysis of left ventricular myocardium in CT angiographic intermediate-degree coronary stenosis improves the diagnostic accuracy for identification of functionally significant stenosis. Eur Radiol. 2019;29:23509.

\section{Figures}




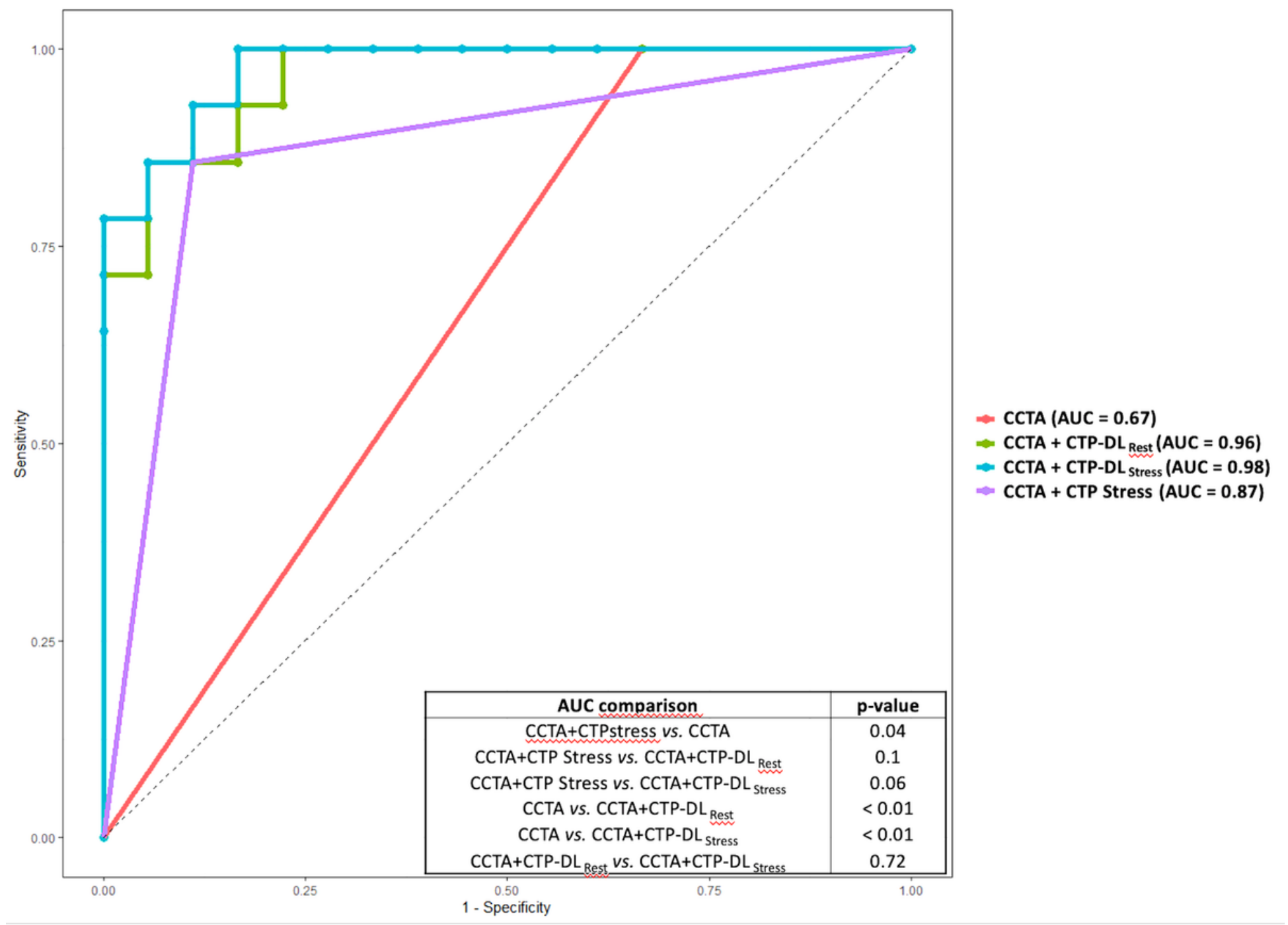

Figure 1

AUC comparisons of the diagnostic performance to detect significant stenosis using CCTA (AUC: $67 \%$ ), CCTA+stress CTP (AUC: 87\%), CCTA+DL rest (AUC: 96\%), CCTA+DL stress (AUC: 98\%). 
340.722

200

150

100

50

0

Human
Prediction Import

\section{Figure 2}

Time comparison for CTP analysis. Left column shows the human time of analysis while the column on the right shows the time of analysis using the CNN approach. The majority of time in the deep learning approach is represented by the importing time. 


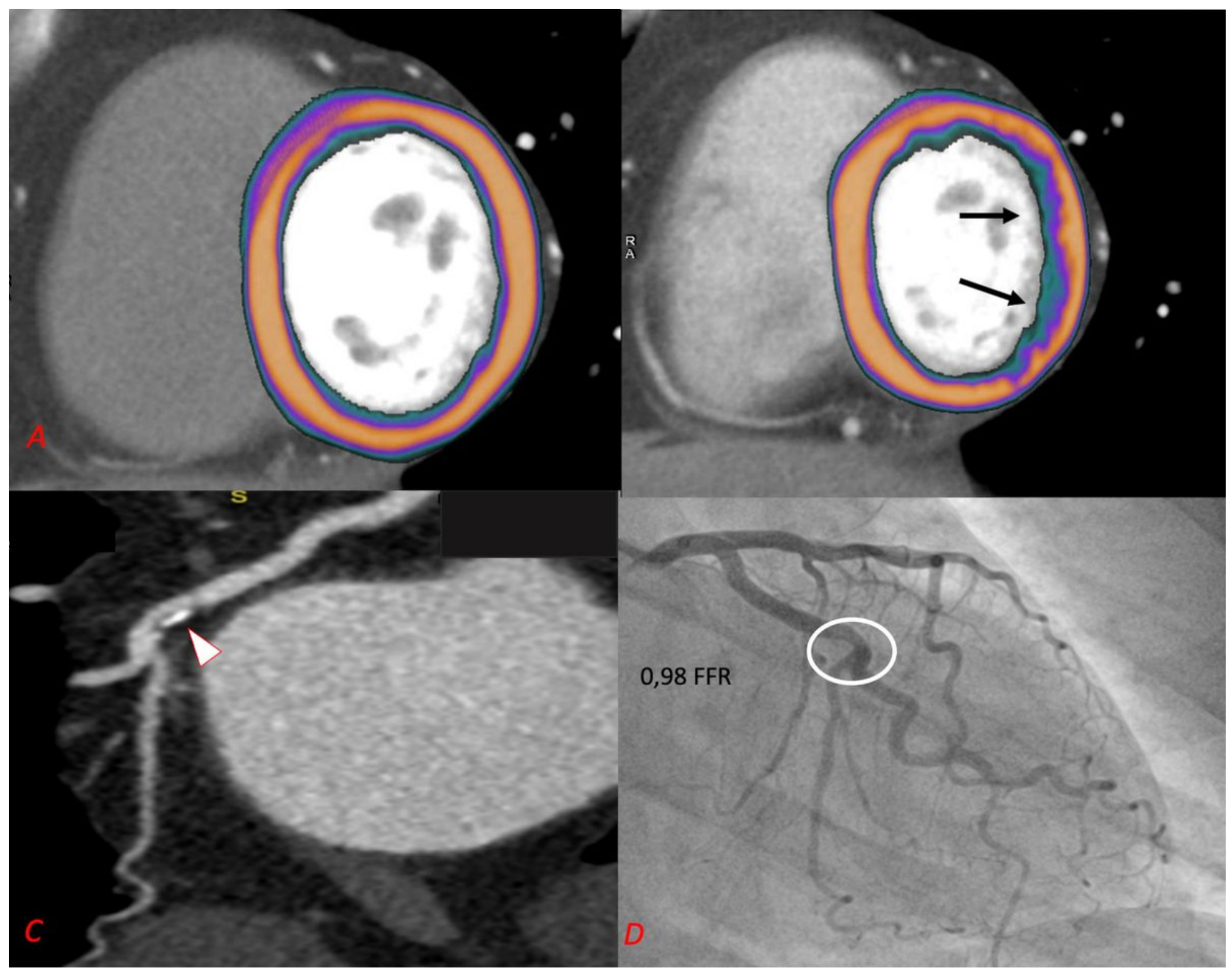

\section{Figure 3}

Seventy-two year-old female patient with hypertension, dyslipidemia, who presented with typical chest pain, and positive SPECT. Normal perfusion was observed in the rest dataset (A) while a mid-ventricle lateral perfusion defect was observed on stress myocardial CTP (arrow, B); CCTA showed moderate stenosis with fibro-calcific plaque in the LCX (arrowhead, C); FFR did not reveal a hemodynamically significant stenosis(d). The prediction of both CTP-DLrest and CTP-DLstress correctly identified the patient with hemodynamically insignificant CAD. CCTA: computed tomography coronary angiography; CTP: computed tomography perfusion; ICA: invasive coronary angiography; FFR: fractional flow reserve; LCX: left circumflex coronary artery; SPECT: single photon emission computed tomography; 


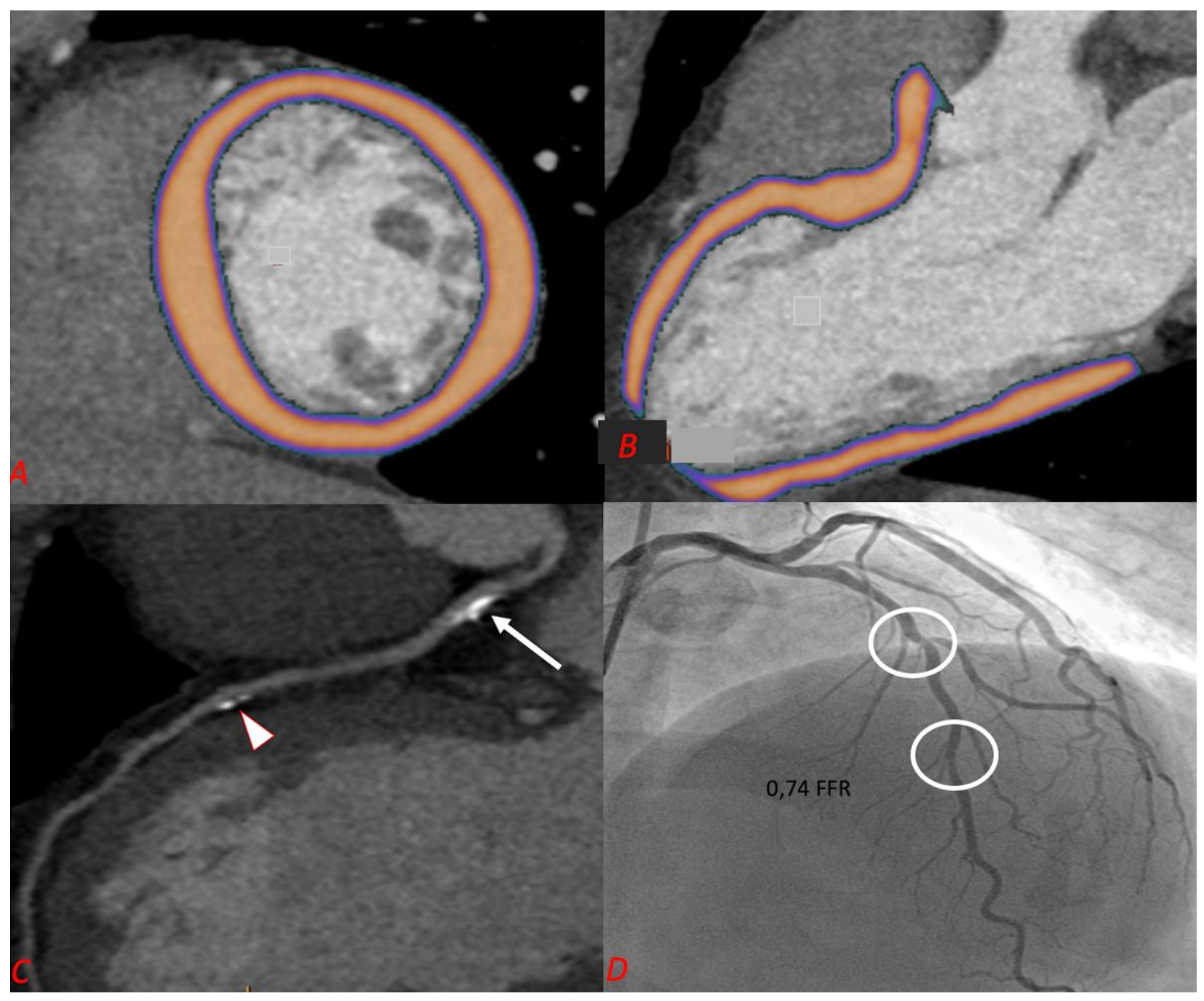

Figure 4

Clinical case showing the additional value of CTP-DLrest over stress CTP to rule out functionally relevant coronary stenoses. 44 year-old male with family history of CAD, smoking, and dyslipidemia presented with severe epigastric pain and a negative stress myocardial CTP (A-B); CCTA showed moderate calcified plaque in the proximal (arrow, C) and middle LAD (arrowhead, C). FFR assessment following ICA demonstrated functionally significant stenosis ( $D, F F R=0.74)$. CTP-DLrest algorithm correctly identified this patient as positive for significant CAD. CAD: coronary artery disease; CTP: computed tomography perfusion; CCTA: coronary computed tomography angiography; FFR: fractional flow reserve; ICA: invasive coronary angiography. 